\title{
PRIMER REGISTRO DE MALEPHORA PURPUROCROCEA (AIZOACEAE, RUSCHIOIDEAE) PARA LA FLORA ARGENTINA
}

\author{
Adriel I. Jocou, Carlos R. Minué \& Ricardo Gandullo
}

Departamento de Biología Aplicada, Facultad de Ciencias Agrarias, Universidad Nacional del Comahue, RN N ${ }^{\circ} 151$ km 12,5, CC 85, CP 8303, Cinco Saltos, Río Negro, Argentina; adrieljocou@gmail.com (autor corresponsal).

\begin{abstract}
Jocou, A. I.; C. R. Minué \& R. Gandullo. 2019. First record of Malephora purpurocrocea (Aizoaceae, Ruschioideae) for the Argentinean Flora. Darwiniana, nueva serie 7(1): 141-151.

The genus Malephora is native to South Africa. Due to its high adaptability, M. purpurocrocea is the most widespread species of the genus, cultivated as ornamental, in arid and semi-arid environments of the Argentinean Patagonia. In this contribution, several spontaneous populations of M. purpurocrocea are reported for the first time for Argentina. The species was observed and collected in different environments in the provinces of Neuquén, Río Negro, and Chubut. A detailed description of the species, distribution and habitat, common names, uses, and a key to the genera of Aizoaceae present in Argentina are included. Due to the lack of detailed studies on the morphology of this species, a series of field photographs and details of microscopic structures are also presented.
\end{abstract}

Keywords. Aizoaceae; Argentinean Flora; Malephora; Patagonia; Ruschioideae.

Resumen. Jocou, A. I.; C. R. Minué \& R. Gandullo. 2019. Primer registro de Malephora purpurocrocea (Aizoaceae, Ruschioideae) para la Flora Argentina. Darwiniana, nueva serie 7(1): 141-151.

El género Malephora es nativo de Sudáfrica. Del total de especies con las que cuenta, $M$. purpurocrocea es la más difundida como ornamental en ambientes áridos y semiáridos de la Patagonia Argentina debido a su alta capacidad de adaptación. En esta contribución se menciona por primera vez a M. purpurocrocea como espontánea en la Argentina. La especie fue observada y recolectada en diversos ambientes de las provincias de Neuquén, Río Negro y Chubut. Se incluyen la descripción detallada de la especie, su distribución y hábitat, nombres vulgares, usos y una clave de los géneros de Aizoaceae presentes en la Argentina. Debido a la escasez de estudios detallados sobre la morfología de esta especie, se presenta una serie de fotografías de campo y detalles de estructuras bajo microscopio estereoscópico.

Palabras clave. Aizoaceae; Flora Argentina; Malephora; Patagonia; Ruschioideae.

\section{INTRODUCCIÓN}

La familia Aizoaceae presenta cerca de 146 géneros, agrupados en cuatro subfamilias: Aizooideae, Mesembryanthemoideae, Ruschioideae y Sesuvioideae (Hartmann, 2017). El género Malephora N.E.Br. es nativo de Sudáfrica, comprende cerca de 17 especies y es el único de la subfamilia Ruschioideae considerado como maleza, que se instala en terrenos bajos y alterados (Hartmann, 2017). Muchas especies de Malephora, en particular M. purpurocrocea (Haw.) Schwantes, son apreciadas como ornamentales por su abundante y prolongada floración (Zangger, 1966; Newman et al., 1981; Hartmann, 2017).

En este trabajo se menciona por primera vez para la Flora Argentina a Malephora purpurocrocea, un sufrútice originario del sur de África y naturalizado en NorteaméricayEuropa(Ferren etal., 1981; GuillotOrtiz 
et al., 2008; Pyke, 2008; Padrón Mederos et al., 2009; Guillot \& Van der Meer, 2010; Sciberras \& Sciberras, 2010 y Hosking et al., 2011), y recientemente hallado como espontáneo en las provincias de Neuquén, Río Negro y Chubut, en la Patagonia Argentina. Se presenta una descripción detallada de la especie, un mapa con la distribución geográfica en Patagonia y una clave para diferenciar a Malephora del resto de los géneros de la familia Aizoaceae reportados en Argentina (Zuloaga et al., 2019). Además, debido a la escasez de estudios detallados sobre la morfología de $M$. purpurocrocea, se presenta una serie de fotografías de campo y de diferentes estructuras (hoja, fruto y semilla) bajo microscopio estereoscópico.

\section{MATERIALES Y MÉTODOS}

Se realizaron diversas observaciones de campo en diferentes localidades de las provincias de Neuquén (Centenario y Neuquén Capital), Río Negro (Cinco Saltos, Ingeniero Huergo, San Antonio Oeste y Sierra Grande) y Chubut (Puerto Madryn, Rawson y Trelew). Para la determinación del material colectado se consultó bibliografía específica (Ferren et al., 1981; Bittrich \& Hartmann, 1988; Hartmann, 1991; Bleck, 2003; Vivrette et al., 2003; Guillot Ortiz et al., 2008; Guillot \& Van der Meer, 2010; Hosking et al., 2011; Hartmann, 2017). El nombre correcto del taxón se verificó a través de la base de datos The International Plant Names Index (http://www.ipni.org). Asimismo, se cotejó el material con una imagen de alta resolución de una ilustración botánica de M. M. Page depositada en el herbario BOL (BOL133919, imagen disponible en: http://www.digitalcollections. lib.uct.ac.za/collection/islandora-24232). Se examinó material adicional de Malephora purpurocrocea y $M$. crocea, a través de imágenes de alta resolución de los herbarios SD y DES (SD152123, SD225964, SD157640, DES00078248), disponibles en Consortium of California Herbaria (http://ucjeps.berkeley. edu/) e ilustraciones científicas depositadas en "Library, Art \& Archives" de "Royal Botanic Gardens", Kew. La descripción de la especie fue realizada a partir de la observación del material fresco. El material de referencia que documenta el trabajo fue depositado en el Herbario Institucional Agronomía Región Comahue (ARC) de la Facultad de Ciencias Agrarias, Universidad Nacional del Comahue (Thiers, 2019).

\section{RESULTADOS}

Malephora purpurocrocea (Haw.) Schwantes, Gartenflora 77: 69. 1928. Mesembryanthemum purpurocroceum Haw., Observ. Gen. Mesembryanthemum (II): 257. 1795. Mesembryanthemum croceum var. purpurocroceum (Haw.) DC., Prodr. (DC.) 3: 438. 1828. Hymenocyclus purpurocroceus (Haw.) L. Bolus, S. African Gard. 17: 399. 1927. Crocanthus purpurocroceus (Haw.) L. Bolus, Fl. Pl. South Africa. 7: tab. 255. 1927. Malephora crocea var. purpurocrocea (Haw.) H. Jacobsen \& Schwantes, Natl. Cact. Succ. J. 13(4): 78. 1958. TIPO: no designado. Figs. 2-6.

Mesembryanthemum insititium Willd., Enum. Pl. (I): 536. 1809. TIPO: no designado.

Sufrútice postrado a suberecto, glabro; matas hasta $30 \mathrm{~cm}$ de alto y 1,5-2(-5) $\mathrm{m}$ de diám. Tallos jóvenes suculentos, verde-azulados y céreos; los adultos leñosos, de color crema a gris claro, débiles, hasta 0,8 cm de diám.; entrenudos de $3-6 \mathrm{~cm}$. Hojas opuestas, suculentas, teretes a subtríquetras, connadas en la base, rectas o levemente curvadas, de $(1,5-) 2-8 \times(0,45-) 0,6-1,1 \mathrm{~cm}$, verde-azuladas hasta rojizas, céreas, mucronadas en el ápice, a veces múticas. Flores solitarias, axilares o terminales, de 2,5-5 cm de diám.; pedicelos de 1-5,5 cm. Sépalos $4-6$, suculentos, desiguales, al menos 2 cortos y 2 largos, los cortos de borde hialino, acuminados, de 8-15 $\times 5-8 \mathrm{~mm}$, los largos semejantes a hojas, de 16-25 $\times$ 8-10 mm. Estaminodios 40-60, petaloides, libres, de bordes lisos, con nervadura central conspicua, lineares, de 13-20 × 2-3 mm, con ápice mucronado o bidentado, a veces obtusos, tornasolados, anaranjados a purpúreos en la cara adaxial y púrpura en la abaxial. Estambres numerosos; filamentos blancos a amarillentos, de 1,5-3 mm, cubiertos de abundantes pelos blancos y cortos en la base; anteras amarillas a anaranjadas, dorsifijas, ligeramente versátiles, de dehiscencia longitudinal, ca. $1,5 \times 0,5 \mathrm{~mm}$. Estigmas 8-11(13), sésiles, triangulares, ovados hasta rómbicos, con abundantes papilas en la cara ventral, de 2-2,5 $\times$ 1,5-2 mm. Ovario obcuneiforme, 8-11(-13) carpelar, placentación parietal. Cápsula loculicida, higrocástica, 8-11(-13) locular, obcuneiforme, de 0,6-1,3 cm de diám.; valvas aladas con quillas 
de expansión de margen irregularmente dentado, parduzcas; membranas de cobertura 2 por lóculo, que cubren desde $2 / 3$ a $3 / 4$ del mismo, con pequeñas máculas blancas dispersas, bordes plegados hacia el interior cuando vacío; cuerpo de cierre presente. Semillas numerosas, obovoides, ca. $1 \times 0,8 \mathrm{~mm}$, oscuras, tuberculadas, con los abultamientos en hileras.

Iconografía. Ferren et al. (1981: 82, fig. 1); Bleck (2003); M. M. Page depositada en el herbario BOL (http://www.digitalcollections.lib.uct.ac.za/ collection/islandora-24232).

Nombres vulgares. En Argentina "rayito de sol" (Dalmasso \& Duplancic, 2018). En países anglosajones "iceplant", “croceum iceplant", "crocea iceplant", "angular pigface" (Zangger, 1966; Newman et al., 1981; Hosking et al., 2011).

Distribución y hábitat. Especie nativa del sur de África, naturalizada en Estados Unidos de América (Ferren et al., 1981; Bleck, 2003), España (Guillot Ortiz et al., 2008; Padrón Mederos et al., 2009; Guillot Ortiz \& Van der Meer, 2010), Australia (Hosking et al., 2011), Malta (Sciberras \& Sciberras, 2010) y México (Bleck, 2003). Malephora purpurocrocea es un caméfito, ocasionalmente saxícola (Sciberras \& Sciberras, 2010), que crece en suelos margosos -ricos en arcilla, calcita y carbonato de calcio- (Hartmann, 2017), ambientes costeros (playas, médanos y acantilados) y alterados (bordes de carreteras, solares, montículos de escombros y cultivos), orillas de ríos y llanuras aluvionales (Ferren et al., 1981; Pyke, 2008; Padrón Mederos et al., 2009; Sciberras \& Sciberras, 2010; Hosking et al., 2011; Hartmann, 2017). Hosking et al. (2011) mencionan que esta especie es un componente predominante en los pastizales del norte de Nueva Gales (Australia) y cohabita con especies nativas.

En la Argentina se han hallado densas poblaciones de Malephora purpurocrocea en 8 localidades pertenecientes a las provincias de Neuquén, Río Negro y Chubut (Fig. 1). En la Tabla 1 se detallan los ambientes en los cuales se desarrolla espontáneamente $M$. purpurocrocea en cada provincia argentina.
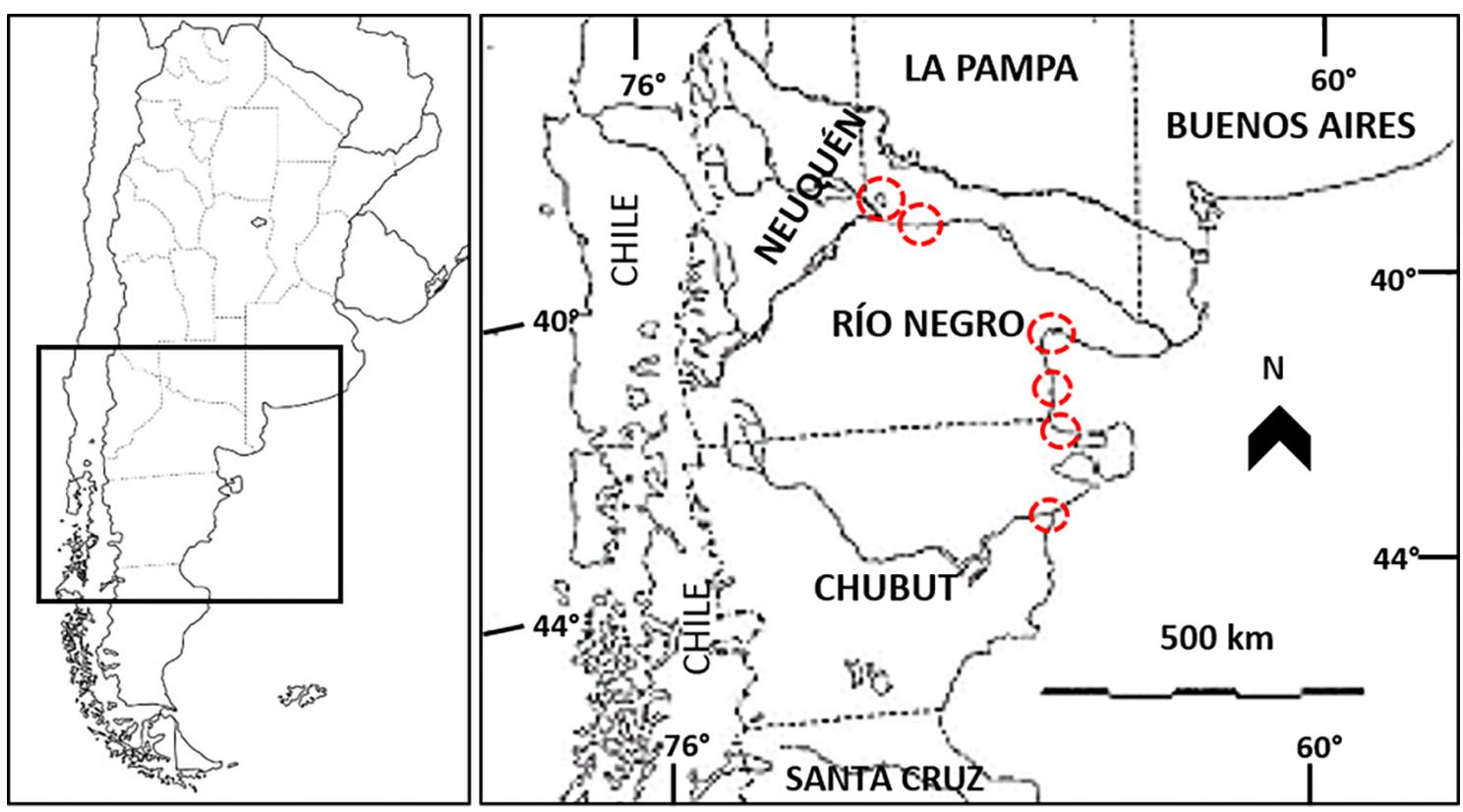

Fig. 1. Distribución de Malephora purpurocrocea en la Argentina. Figura en color en la versión en línea http://www. ojs.darwin.edu.ar/index.php/darwiniana/article/view/821/1161 
Tabla 1. Localidades donde se estudiaron poblaciones espontáneas de Malephora purpurocrocea, características de cada ambiente y elementos florísticos dominantes.

\begin{tabular}{|c|c|c|c|}
\hline Provincia & Localidad & Zona & Características \\
\hline \multirow{2}{*}{ Neuquén } & Neuquén Capital & \multirow[b]{2}{*}{ - } & \multirow{2}{*}{ Sobre laderas barrancosas en cercanías a áreas urbanizadas. } \\
\hline & Centenario & & \\
\hline \multirow{4}{*}{ Río Negro } & Cinco Saltos & $\begin{array}{l}\text { Inmediaciones del } \\
\text { Lago Pellegrini }\end{array}$ & $\begin{array}{l}\text { Sobre suelos arcillosos y pedregosos, en bajos salinos con régimen } \\
\text { hídrico temporal a causa de las fluctuaciones características de este } \\
\text { cuerpo de agua artificial (Amalfi, 2009). Cohabita con especies } \\
\text { características de ambientes salinos, coincidente con lo observado por } \\
\text { Ferren et al. (1981), como Atriplex undulata (Moq.) D. Dietr., Baccharis } \\
\text { spartioides (Hook. \& Arn. ex DC.) J. Remy, Baccharis tenella Hook. } \\
\text { \& Arn., Bassia scoparia (L.) A.J. Scott, Distichlis spicata (L.) Greene } \\
\text { var. spicata, Sarcocornia neei (Lag.) M.A. Alonso \& M.B. Crespo y } \\
\text { Tamarix ramosissima Ledeb. }\end{array}$ \\
\hline & \multirow[t]{2}{*}{$\begin{array}{l}\text { San Antonio } \\
\text { Oeste }\end{array}$} & $\begin{array}{l}\text { Ciudad de San } \\
\text { Antonio Oeste }\end{array}$ & $\begin{array}{l}\text { Sobre terrenos alterados en cercanías al mar. Cohabita con especies } \\
\text { típicas de la Región del Monte como Atriplex undulata, Grindelia } \\
\text { chiloensis (Cornel.) Cabrera y diferentes especies de coirones (pastos } \\
\text { duros típicos de la Patagonia) pertenecientes a los géneros Poa, } \\
\text { Pappostipa, Nassella, Jarava y Pappophorum. }\end{array}$ \\
\hline & & $\begin{array}{l}\text { Balneario Las } \\
\quad \text { Grutas }\end{array}$ & $\begin{array}{l}\text { En acantilados costeros. Cohabita con Carpobrotus sp., Grindelia } \\
\text { chiloensis, Mesembryanthemum nodiflorum L., Sporobolus rigens } \\
\text { (Trin.) E. Desv. y Tamarix ramosissima. }\end{array}$ \\
\hline & Ingeniero Huergo & $\begin{array}{l}\text { Entre zonas de } \\
\text { Monte y urbanas }\end{array}$ & $\begin{array}{l}\text { En suelos arenosos alterados cercanos a laderas barrancosas, en } \\
\text { cercanías a áreas urbanizadas. Junto a Atriplex lampa (Moq.) D. Dietr. }\end{array}$ \\
\hline \multirow{3}{*}{ Chubut } & Puerto Madryn & $\begin{array}{l}\text { Lobería de Punta } \\
\text { Loma }\end{array}$ & Cohabita junto a especies de Monte, sobre acantilados. \\
\hline & Rawson & $\begin{array}{l}\text { Balneario Playa } \\
\text { Unión }\end{array}$ & $\begin{array}{l}\text { En terrenos fuertemente alterados y bajos, con suelo arenoso y sobre } \\
\text { la línea costera. Cohabita con Carpobrotus sp., Mesembryanthemum } \\
\text { nodiflorum, Sporobolus rigens y Suaeda argentinensis A. Soriano. }\end{array}$ \\
\hline & Trelew & - & $\begin{array}{l}\text { En un establecimiento abandonado (Magdalena Llorens, comunicación } \\
\text { personal). }\end{array}$ \\
\hline
\end{tabular}

Las observaciones sugieren que Malephora purpurocrocea se desarrolla favorablemente en suelos salinos, pedregosos, de texturas arenosas a arcillosas, con tolerancia a la escasa precipitación, temperaturas máximas cercanas a $40^{\circ} \mathrm{C}$ y mínimas a $-10^{\circ} \mathrm{C}$; en concordancia con los resultados obtenidos por Dalmasso \& Duplancic (2018).

Usos. Malephora purpurocrocea es una especie cultivada como ornamental en regiones con escasa precipitación y temperaturas extremas, por su alta rusticidad, abundante y extensa floración (Zangger, 1966; Ferren et al., 1981; Newman et al., 1981; Bleck, 2003; Guillot Ortiz et al., 2008;
Padrón Mederos et al., 2009; Hartmann, 2017; Dalmasso \& Duplancic, 2018). Ha sido utilizada para control de la erosión del suelo y para retardar el avance del fuego en los incendios (Zangger, 1966; Newman et al., 1981).

Observaciones. Malephora purpurocrocea es una especie afín a $M$. crocea (Jacq.) Schwantes, con la que suele confundirse con facilidad. Las descripciones de una u otra especie realizadas por Haworth (1795), Jacquin (1809), Willdenow (1809), De Candolle (1828) y Hartmann (2017), permiten, hasta el momento, concluir que se diferencian en que $M$. purpurocrocea posee la 
cara abaxial de los estaminodios con al menos una ligera coloración púrpura, mientras que $M$. crocea es completamente naranja, con alguna tonalidad rojiza en el centro de la flor. Se observó a campo que, dentro de un mismo espécimen, la coloración de la cara adaxial de los estaminodios de $M$. purpurocrocea es altamente variable (naranja, rojo, púrpura, rosa); mientras que la coloración de la cara abaxial se mantuvo constante en tonalidades púrpuras. Además, en las poblaciones de Playa Unión, Las Grutas e Ingeniero Huergo se hallaron ejemplares aberrantes (Jocou \& Minué 2048 ARC; Jocou, Minué \& Gandullo 2184
ARC) en baja frecuencia con la cara abaxial de los estaminodios suavemente coloreados de rosa, casi imperceptible y enmascarado por el naranja (Fig. 3D-E). Esta alteración en la coloración de $M$. purpurocrocea presta a confusión para diferenciarla de $M$. crocea.

Ferren et al. (1981) distinguen erróneamente a Malephora purpurocrocea de $M$. crocea a través de la coloración de la cara adaxial de los estaminodios, carácter reportado como muy variable en el presente estudio, por lo que la descripción y observaciones que realizan los autores mencionados corresponden a $M$. purpurocrocea.
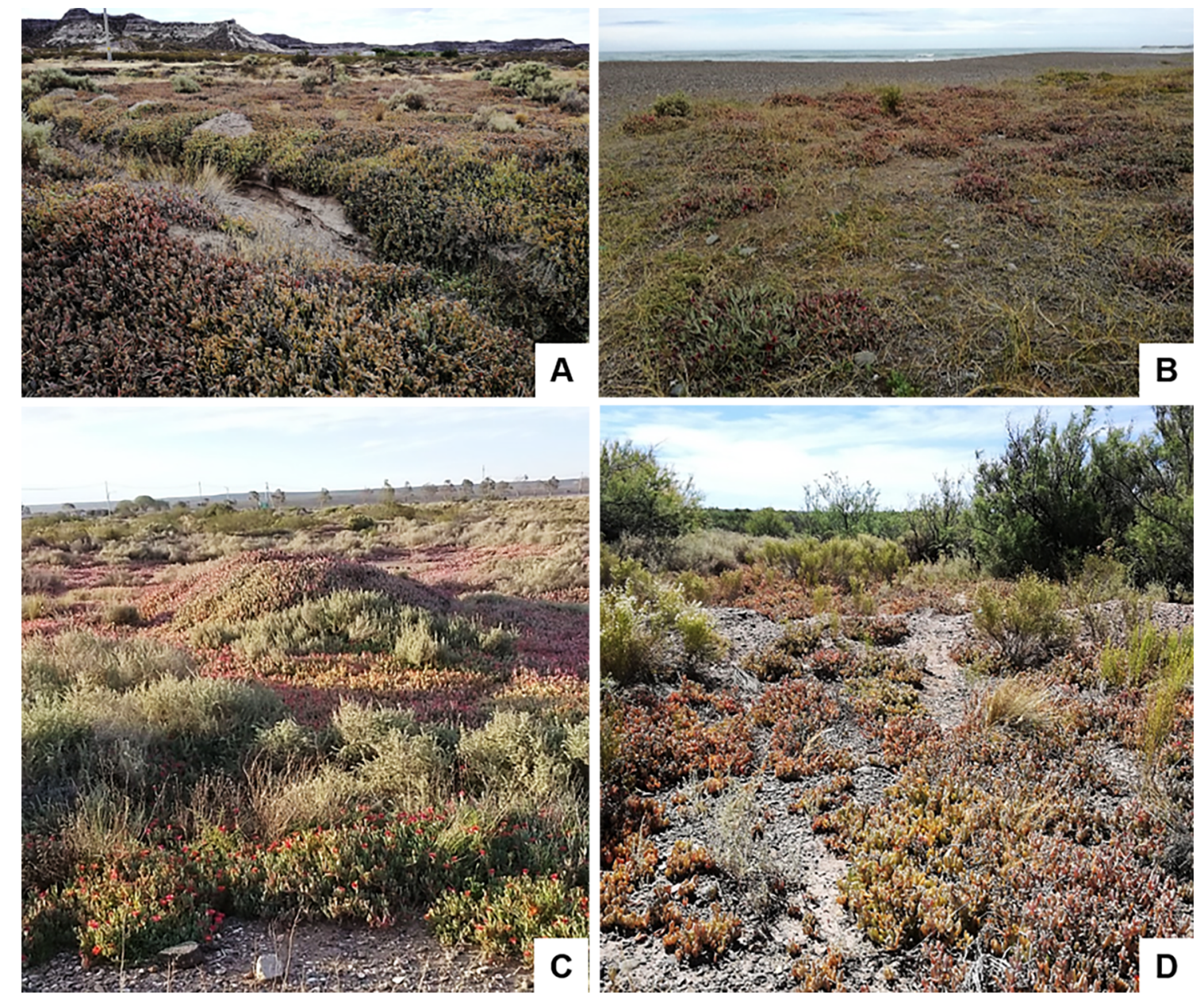

Fig. 2. Malephora purpurocrocea. Vista general de poblaciones. A, en Ingeniero Huergo junto a especies del Monte. B, en la costa del balneario Playa Unión, Rawson. C, en un terreno alterado de San Antonio Oeste. D, en cercanías a zonas urbanizadas del Lago Pellegrini, nótese el suelo pedregoso. Fotos A, B y D de Adriel Jocou, foto C de Arian Jocou. Figura en color en la versión en línea http://www.ojs.darwin.edu.ar/index.php/darwiniana/article/view/821/1161 

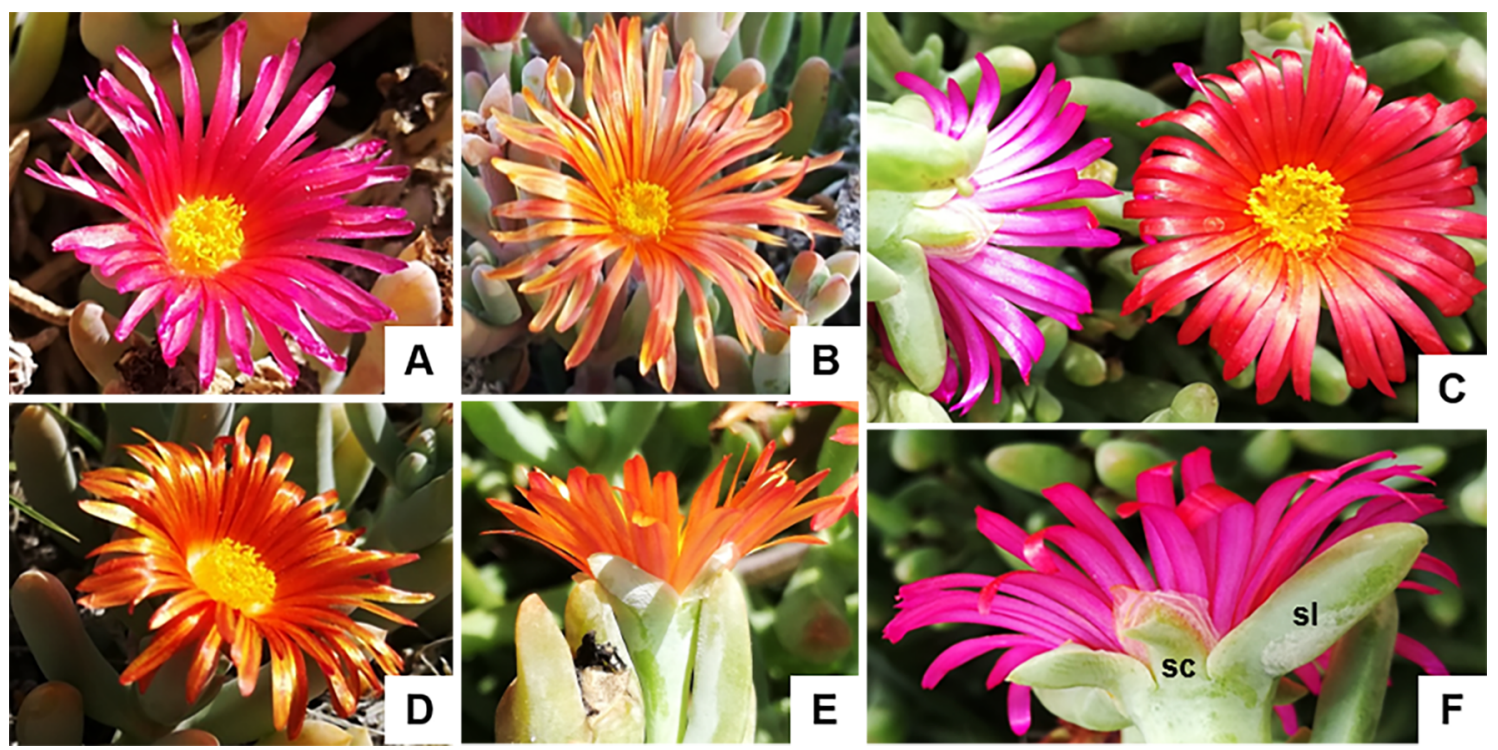

Fig. 3. Malephora purpurocrocea. Morfología y diversidad floral. A, ejemplar naturalizado en Lago Pellegrini, Cinco Saltos. B, ejemplar naturalizado en San Antonio Oeste, nótese la vena media conspicua. C, contraste de coloraciones entre la cara adaxial y abaxial de los estaminodios en ejemplares normales. D, E, contraste de coloraciones entre la cara adaxial y abaxial de los estaminodios en ejemplares aberrantes. F, detalle del cáliz: sépalo corto (sc) y sépalo largo (sl). Fotos A, C-F de Adriel Jocou, foto B de Arian Jocou. Figura en color en la versión en línea http://www.ojs.darwin.edu.ar/index.php/darwiniana/article/view/821/1161

Esto condujo a la reiteración del error por Bleck (2003) y Hosking et al. (2011), al basarse en Ferren et al. (1981); y posteriormente, por Padrón Mederos et al. (2009) al basarse en Bleck (2003). Por este motivo, es conveniente tratar al taxón reportado por ellos como $M$. purpurocrocea.

Las observaciones de campo indican que $M a$ lephora purpurocrocea presenta variabilidad en cuanto a las dimensiones foliares y cantidad de flores según la disponibilidad de agua, tal como lo señalan Dalmasso \& Duplancic (2018). Por otro lado, la coloración de las hojas puede variar desde tonalidades verde-azuladas hasta rojizo-violáceas según un mayor o menor aporte de agua, respectivamente, como así también a causa de los fríos invernales.

Según lo observado, Malephora purpurocrocea posee ombrohidrocoria como mecanismo de dispersión primario y bitisocoria ('Bythisochory') como secundario, lo cual es coincidente con lo señalado para diversos géneros de Aizoaceae (Parolin, 2006). La morfología particular de las cápsulas higrocásticas permite que, durante la lluvia, se activen los mecanismos de apertura. La energía cinética de las gotas de agua que impactan sobre las membranas de cobertura permite expulsar las semillas del fruto, proceso regulado por los cuerpos de cierre. Las semillas son luego transportadas por los pequeños cursos de agua formados durante las lluvias. En la Fig. 4 se evidencia el crecimiento de plántulas de $M$. purpurocrocea a lo largo de pequeños surcos naturales formados por las precipitaciones.

Las poblaciones halladas durante la investigación alcanzan a cubrir el suelo hasta en un $80 \%$, presentan muy buen desarrollo y evidencias de continuidad en la dispersión. Según registros propios, las poblaciones se autoperpetúan sin intervención del hombre desde hace al menos 10 años. Al considerarse también la distancia entre poblaciones (ca. de $600 \mathrm{~km}$ entre localidades y provincias diferentes), se demuestra la naturalización de Malephora purpurocrocea en Argentina, según los criterios postulados por Pyšek et al. (2004). 

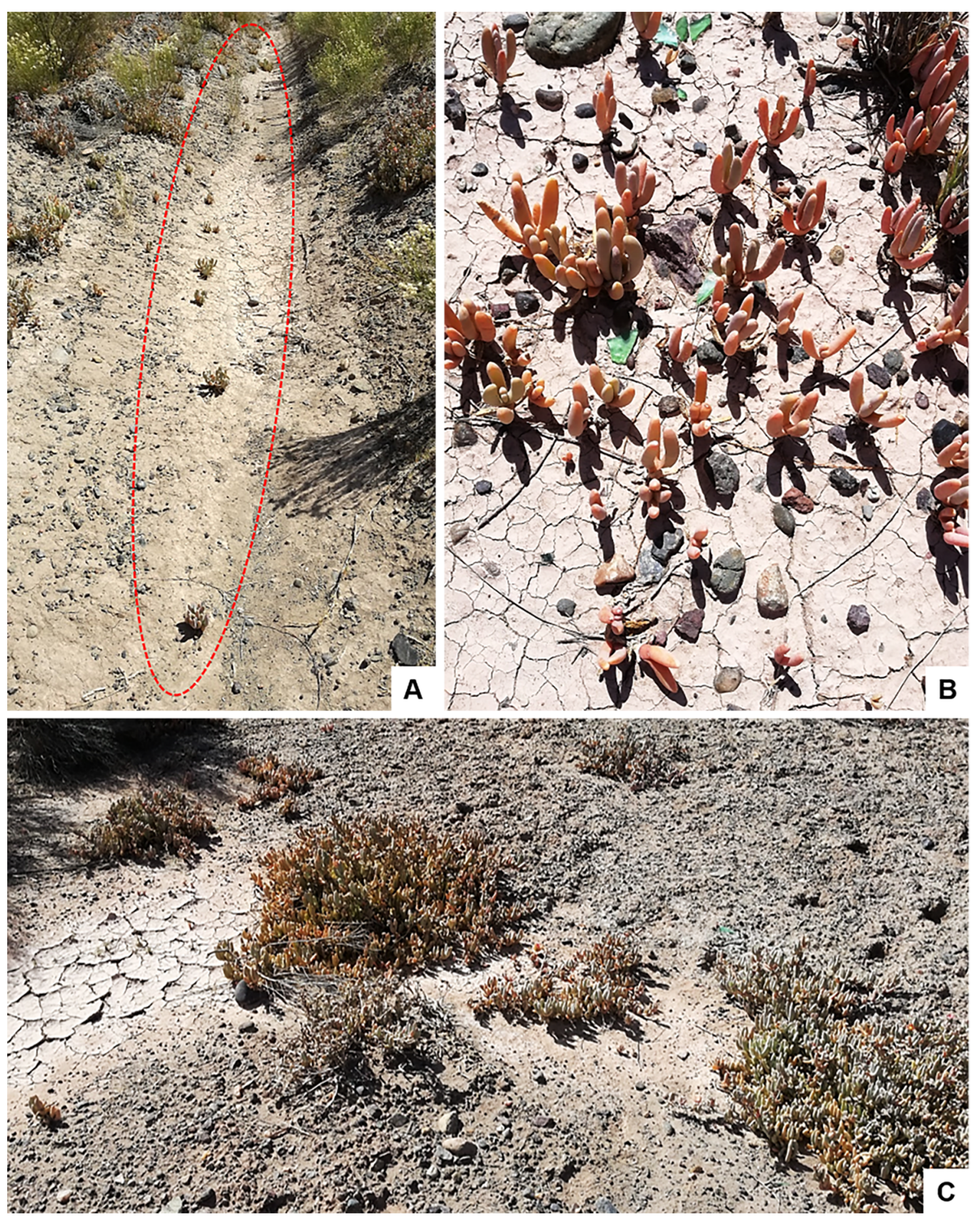

Fig. 4. Malephora purpurocrocea. Poblaciones en cercanías al Lago Pellegrini, Cinco Saltos. A, evidencia de bitisocoria, crecimiento de plántulas en un pequeño surco formado por las lluvias. $\mathbf{B}$, detalle de plántulas sobre una pequeña zona aluvial. C, vista general del desarrollo en zonas afectadas por las precipitaciones. Fotos A-C de Adriel Jocou. Figura en color en la versión en línea http://www.ojs.darwin.edu.ar/index.php/darwiniana/article/view/821/1161 


\section{Material representativo examinado}

ARGENTINA. Chubut. Depto. Rawson, Rawson, Playa Unión, 22-IX-2018, (fl, fr), Jocou \& Minué 2046 (ARC); Playa Unión, 22-IX-2018, (fl, fr), Jocou \& Minué 2047 (ARC); Playa Unión, 22-IX-2018, (fl, fr), Jocou \& Minué 2048 (ARC). Neuquén. Depto. Confluencia, Neuquén, 20-XII-2018, Jocou, Minué \& Gandullo 2049 (ARC). Río Negro. Depto. General Roca, Cinco Saltos, Lago Pellegrini, 10-XII-2012, (fr), Gandullo \& Gimenez GR733 (ARC); Cinco Saltos, 07-I-2018, (fr), Jocou \& Minué 2045 (ARC); Ingeniero Huergo, frente al cementerio, 15-III-2019, (fl, fr), Jocou, Minué \& Gandullo 2183 (ARC); Ingeniero Huergo, frente al cementerio, 15-III-2019, (fl, fr), Jocou, Minué \& Gandullo 2184 (ARC). Depto. San Antonio Oeste, San Antonio Oeste, 20-XII-2013, (fl, fr), Jocou 2044 (ARC); San Antonio Oeste, 13-X2018, (fl, fr), Jocou \& Minué 2050 (ARC).
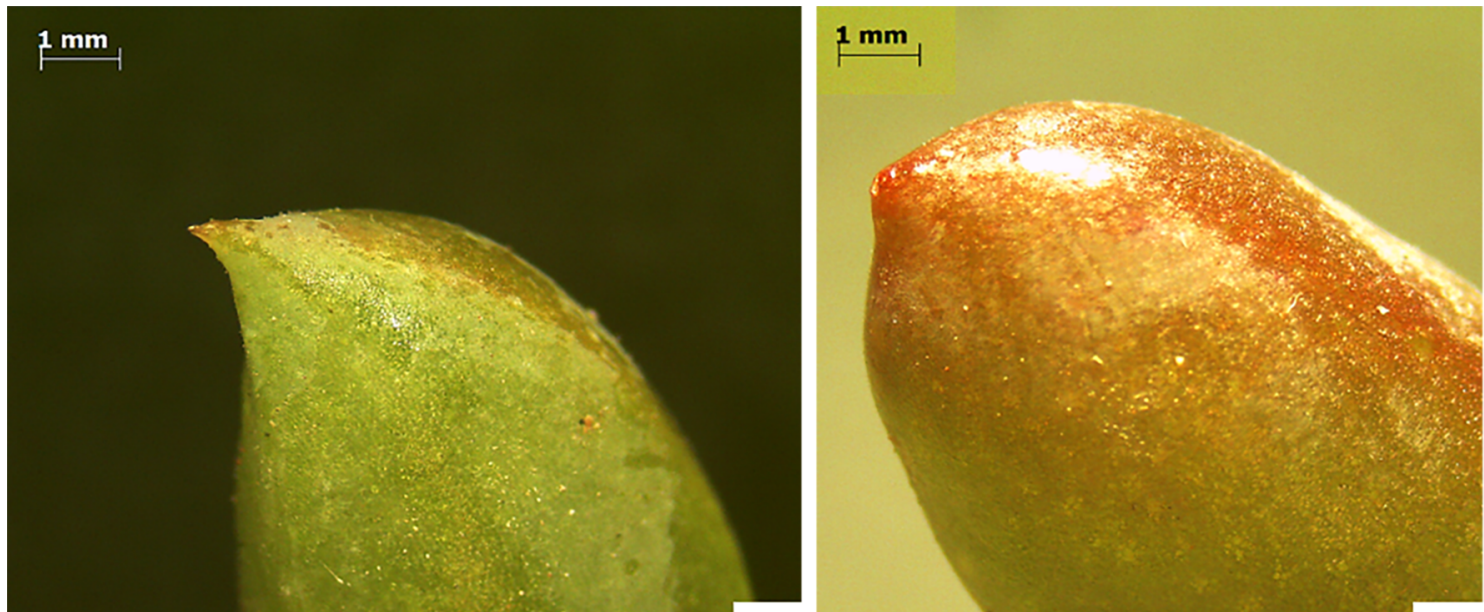

A
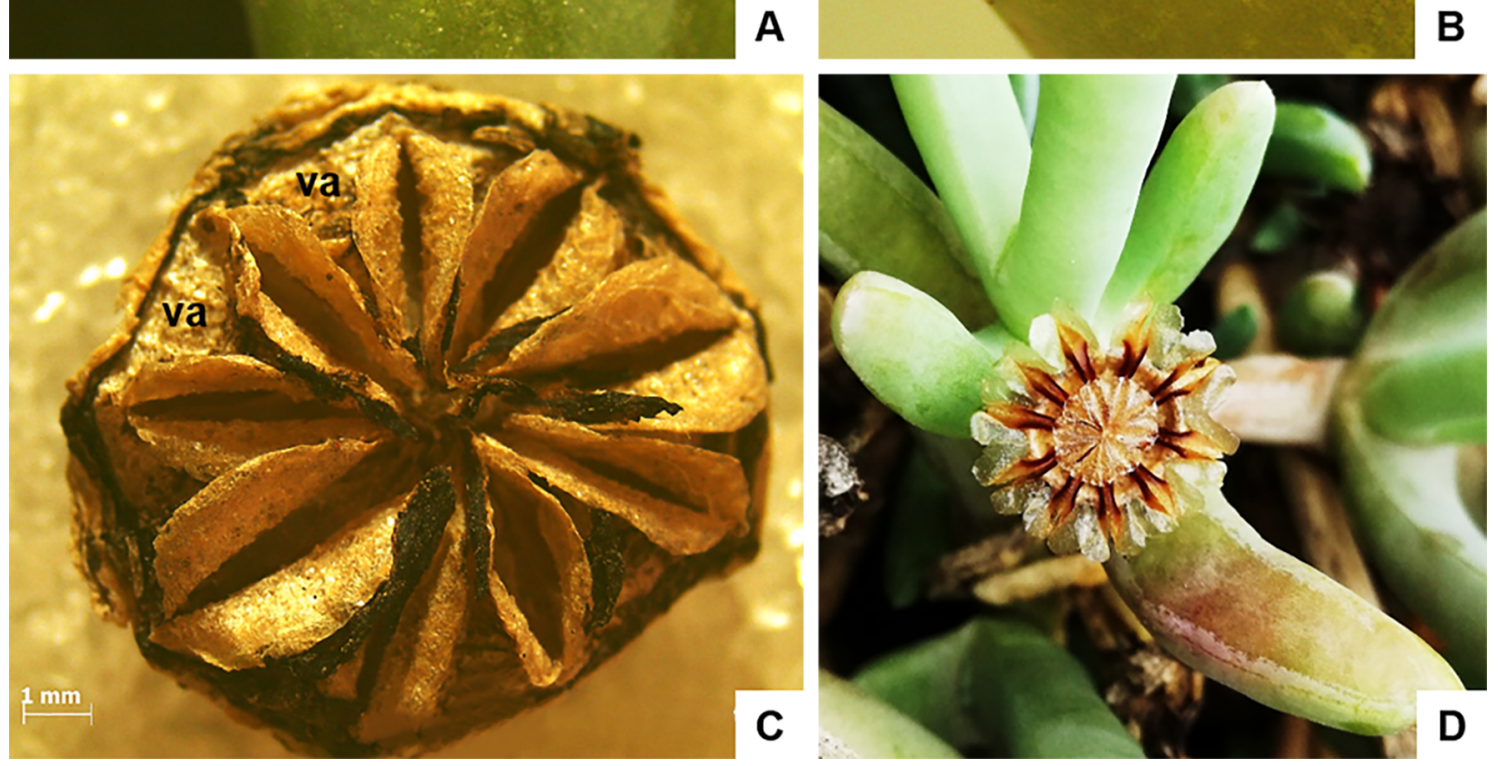

Fig. 5. Malephora purpurocrocea. Hojas y frutos. A, detalle de ápice foliar mucronado. B, detalle de ápice foliar mútico. C, Fruto "ex situ", cerrado, nótense las valvas (va). D, fruto "in situ", abierto debido a las precipitaciones (Rawson). Fotos A-D de Adriel Jocou. Figura en color en la versión en línea http://www.ojs.darwin.edu.ar/index. $\mathrm{php} /$ darwiniana/article/view/821/1161 

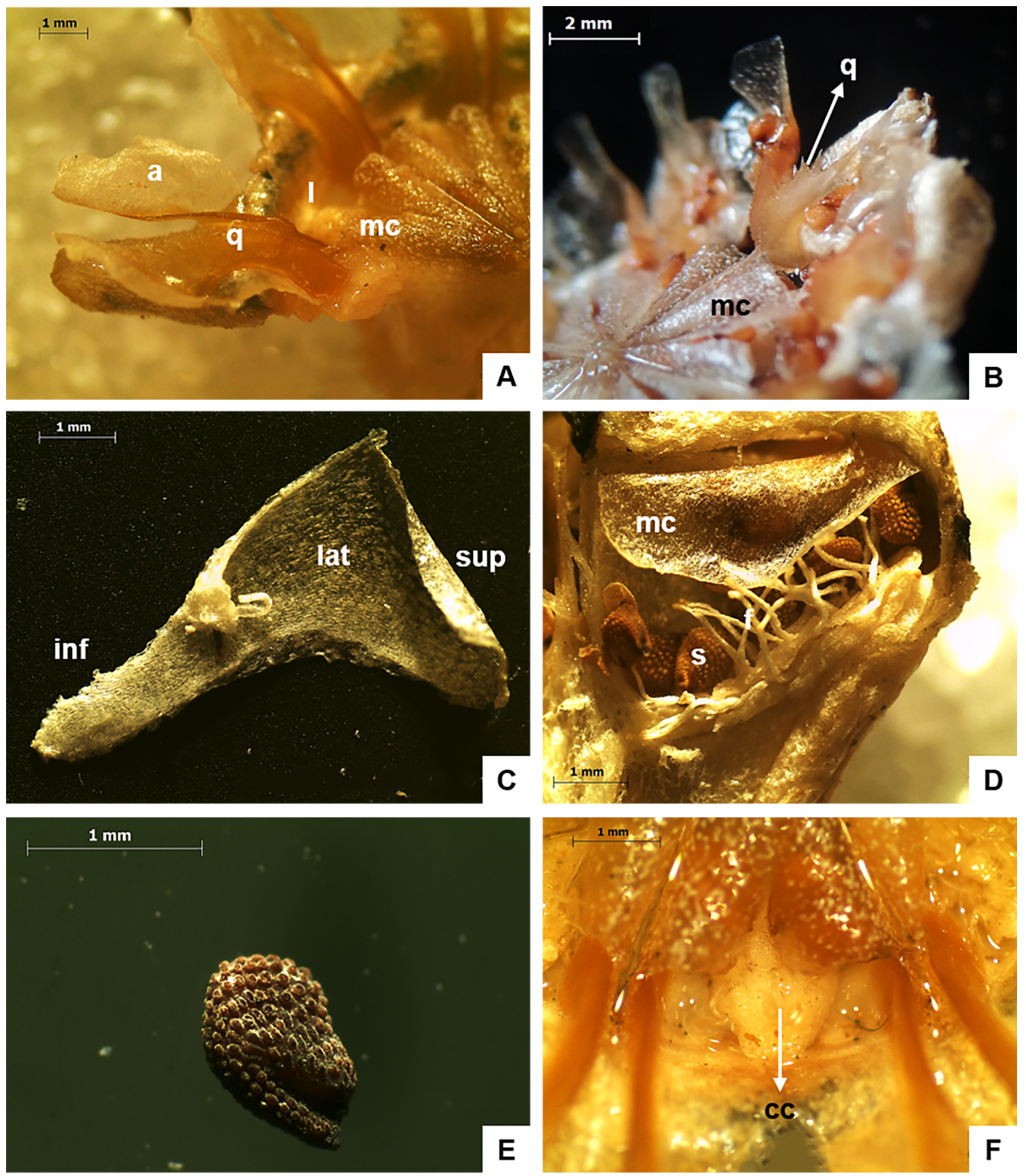

Fig. 6. Malephora purpurocrocea. Fruto y semilla. A, vista general de estructuras principales: membranas de cobertura (mc), quillas de expansión (q), alas de las valvas (a) y lóculo (l). B, detalle del borde irregularmente dentado de la quilla (q), véanse las membranas de cobertura (mc). C, detalle de una membrana de cobertura completa, parte superior (sup), lateral interna (lat) e inferior (inf). D, corte transversal, detalle del lóculo: membrana de cobertura (mc), semillas (s) y funículos (f), nótese la placentación parietal. E, detalle de una semilla, nótese la superficie con tubérculos en hileras. F, detalle del cuerpo de cierre (cc) en la parte libre del lóculo. Fotos A-F de Adriel Jocou. Figura en color en la versión en línea http://www.ojs.darwin.edu.ar/ index.php/darwiniana/article/view/821/1161 


\section{Clave para la identificación de los géneros de Aizoaceae en Argentina}

1. Estaminodios petaloides presentes 2

1. Estaminodios petaloides ausentes

2(1). Placentación central; estaminodios petaloides a veces unidos en la base formando un pequeño tubo (Mesembryanthemoideae) 3

2. Placentación parietal; estaminodios petaloides mayormente libres (Ruschioideae) 4

3(2). Plantas anuales, cubiertas de células vesiculares brillosas, visibles a simple vista; flores en general blancas o crema Mesembryanthemum

3. Plantas perennes, desprovistas de células vesiculares; flores generalmente rosadas Aptenia 4(2). Fruto indehiscente, carnoso; plantas rastreras; hojas notablemente tríquetras Carpobrotus 4. Fruto dehiscente, seco; plantas postradas a suberectas; hojas teretes a subtríquetras, nunca marcadamente tríquetras Malephora

5(1). Segmentos del perianto provistos de apéndices subapicales; fruto cápsula de dehiscencia circuncisa (Sesuvioideae) ....... 6 5. Segmentos del perianto desprovistos de apéndices subapicales; fruto drupáceo, provisto de 4 ornamentaciones en forma de cuerno (Aizooideae) Tetragonia 6(5). Estilo 1 Trianthema

6. Estilos 2-5 Sesuvium

7(6). Frutos maduros completamente septados Cypselea

7. Frutos maduros nunca completamente septados o septos ausentes

\section{AGRADECIMIENTOS}

A los revisores anónimos de Darwiniana nueva serie por sus valiosas sugerencias, que permitieron mejorar el manuscrito. A Gabriel Bernardello por sus comentarios respecto a la taxonomía y nomenclatura. A Cornelia Klak (University of Cape Town, Bolus Herbarium) por facilitar información necesaria para esta investigación. A Julia Buckley (Royal Botanic Gardens, Kew) por proveer las imágenes e información necesaria respecto a ilustraciones del género Malephora. A Michael Berger (Technische Universitaet Berlin, Universitätsbibliothek) y Petra Koch (Freie Universität Berlin, Botanischer Garten und Botanisches Museum, Bibliothek) por proporcionar parte de la bibliografía consultada. A Arian Jocou por facilitar las fotografías $2 \mathrm{C}$ y $3 \mathrm{~B}$.

\section{BIBLIOGRAFÍA}

Amalfi, M. 2009. Lago Pellegrini. Características limnológicas. ProBiota, Museo de La Plata, Facultad de Ciencias Naturales y Museo, UNLP. Series de documentos 7: 1-58.

Bittrich, V. \& H. E. K. Hartmann. 1988. The Aizoaceae - a new approach. Botanical Journal of the Linnean Society 97: 239254. DOI: 10.1111/j.1095-8339.1988.tb01581.x
Bleck, J. E. 2003. Malephora, en Flora of North America Editorial Committee (Eds.), Flora of North America 4: 8990. Disponible en: eFloras.org (Consulta: noviembre 2018).

Dalmasso, A. \& A. Duplancic. 2018. Malephora crocea var. purpureo-crocea (Haw.) Jacobsen \& Schwantes (Aizoaceae) exótica de valor en xerijardinería. Multequina 27: 35-39.

De Candolle, A. P. 1828. Prodromus systematis naturalis regni vegetabilis, vol. 3. Paris: Treuttel et Würtz.

Ferren, W. R.; J. Bleck \& N. Vivrette. 1981. Malephora crocea (Aizoaceae) naturalized in California. Madroño 28: 8085 .

Guillot Ortiz, D.; E. Laguna Lumbreras \& S. A. Roselló Picornell. 2008. Flora alóctona suculenta valenciana: Aizoaceae y Portulacaceae. Monografias de la revista Bouteloua 7: 1-68.

Guillot Ortiz, D. \& P. Van der Meer. 2010. Nuevos taxones vegetales alóctonos de jardinería en el área continental de NE de España: comportamiento e historia. Management of Biological Invasions 1: 6-12. DOI: 10.3391/mbi.2010.1.1.03

Hartmann, H. E. K. (Ed.). 2017. Aizoaceae. Illustrated Handbook of Succulent Plants, $2^{\text {a }}$ edición. Berlin: Springer. DOI: 10.1007/978-3-662-49260-4

Hartmann, H. E. K. 1991. Keys to the genera of Mesembryanthema. South African Journal of Botany 57: 95-106. DOI: 10.1016/S0254-6299(16)30967-X

Haworth, A. H. 1795. Observations on the genus Mesembryanthemum in two parts, parte 2. London: The author. 


\section{A. I. JOCOU ET AL. Malephora purpurocrocea en Argentina}

Hosking, J. R.; B. J. Conn, B. J. Lepschi \& C. H. Barker. 2011. Plant species first recognised as naturalised or naturalising for New South Wales in 2004 and 2005. Cunninghamia 12: 87-114.

Jacquin, N. J. 1809. Fragmenta botanica, figuris coloratis illustrata: ab anno 1800 ad annum 1809 per sex fasciculos edita. Viena: Typis Mathiae Andreae Schmidt, typogr. Universit. DOI: 10.5962/bhl.title.538

Newman, S. R.; B. Meacham \& A. L. Riley. 1981. Plants for California landscapes: a catalog of drought-tolerant plants. Bulletin of California Departament of Water Resources 209: 1-127. DOI: 10.5962/bhl.title.34872

Padrón Mederos, M. A.; I. R. Guma, A. Santos Guerra \& J. A. Reyes Betancort. 2009. Apuntes florísticos y taxonómicos para la flora de las Islas Canarias. Acta Botánica Malacitana 34: 242-251.

Parolin, P. 2006. Ombrohydrochory: Rain-operated seed dispersal in plants - With special regard to jet-action dispersal in Aizoaceae. Flora: Morphology, Distribution, Functional Ecology of Plants 201: 511-518. DOI: 10.1016/j.flora.2005.11.003

Pyke, S. 2008. Contribución al conocimiento de la flora alóctona catalana. Collectanea Botanica 27: 95-104. DOI: 10.3989/ collectbot.2008.v27.8

Pyšek, P.; D. M. Richardson, M. Rejmánek, G. L. Webster, M. Williamson \& J. Kirschner. 2004. Alien plants in checklists and floras: towards better communication between taxonomists and ecologists. Taxon 53: 131-143. DOI: 10.2307/4135498

Sciberras, J. \& A. Sciberras. 2010. A Contribution to the Knowledge of Alien Flora in the Maltese Islands. Central Mediterranean Naturalist 5: 44-48.

Thiers, B. [permanentemente actualizado, consulta 2019] Index Herbariorum: a global directory of public herbaria and associated staff. New York Botanical Garden's Virtual Herbarium, http://sweetgum.nybg.org/science/ih

Vivrette, N. J.; J. E. Bleck \& W. R. Ferren Jr. 2003. Aizoaceae, en Flora of North America Editorial Committee (Eds.), Flora of North America 4: 75-90. Disponible en: eFloras.org (Consulta: noviembre 2018).

Willdenow, C. L. 1809. Enumeratio plantarum horti regii botanici berolinensis, continens descriptiones omnium vegetabilium in horto dicto cultorum, vol. 1. Berlin: Taberna Libraria Scholae Realis.

Zangger, C. 1966. Iceplants as groundcover. Lasca Leaves 1618: 49-53.

Zuloaga, F.; O. Morrone \& M. J. Belgrano. Catálogo de las plantas del Cono Sur. Base de datos permanentemente actualizada y disponible en: http://www2.darwin.edu.ar/ Proyectos/FloraArgentina/fa.htm (Consulta: enero 2019). 